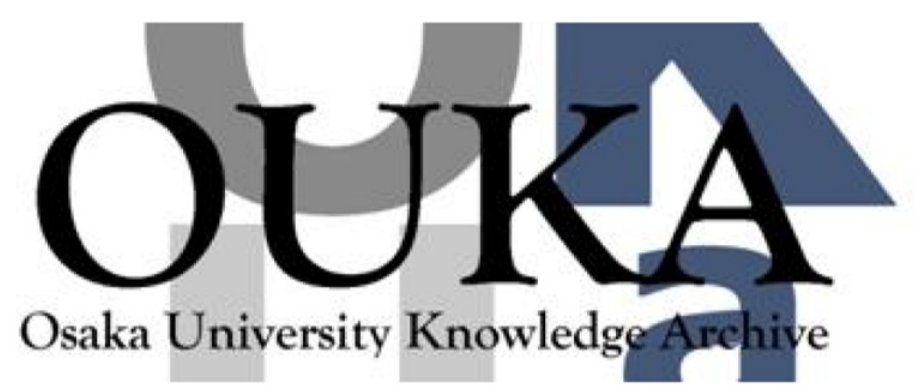

\begin{tabular}{|c|l|}
\hline Title & Laser action in conducting polymers \\
\hline Author(s) & $\begin{array}{l}\text { Frolov, Sergey V. ; Shkunov, Maxim N. ; Vardeny, } \\
\text { Z. Valy et al. }\end{array}$ \\
\hline Citation & $\begin{array}{l}\text { Proceedings of SPIE - The International Society } \\
\text { for Optical Eng ineering. 3145 p. 2-p. 12 }\end{array}$ \\
\hline Issue Date & $1997-12-01$ \\
\hline oaire:version & VoR \\
\hline URL & https://hdl. handle. net/11094/76952 \\
\hline rights & \\
\hline Note & \\
\hline
\end{tabular}

Osaka University Knowledge Archive : OUKA

https://ir. Library. osaka-u. ac. jp/

Osaka University 


\title{
Laser Action in Conducting Polymers
}

\author{
S.V.Frolov ${ }^{\mathrm{a}}$, M.Shkunov ${ }^{\mathrm{a}}$, Z.V.Vardeny ${ }^{\mathrm{a}}$, M.Ozaki ${ }^{\mathrm{b}}$, and K.Yoshino

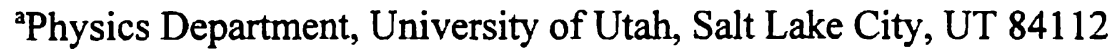 \\ ${ }^{b}$ Department of Electronic Engineering, Faculty of Engineering, Osaka University, Osaka 565, Yamada- \\ Oka 2-1 Suita, Japan
}

\begin{abstract}
We discuss both cooperative radiation and stimulated emission and consider their role in spectral narrowing of luminescent conducting polymers. We argue that cooperative radiation is favored in films with poor optical confinement. On the other hand, directional stimulated emission can be observed in dilute solution and thin films with superior optical confinement. Spectral narrowing in this case can be achieved by increasing either the excitation length or excitation intensity. The optical gain and loss coefficients are measured. Narrow line $\left(\sim 1.5 \mathrm{~cm}^{-1}\right)$ laser emission is observed in cylindrical microcavities formed by thin polymer films coated around glass fibers in the red and green spectral ranges. The cavity quality factors of these plastic lasers are mainly determined by selfabsorption and estimated to be about 5000 .
\end{abstract}

Keywords: conducting polymers, PPV, disubstituted polyacetylene, excitons, spectral narrowing, plastic lasers, stimulated emission, superfluorescence, microcavity lasers.

\section{INTRODUCTION}

Recently dramatic emission spectral narrowing (SN) has been reported in thin films of various luminescent conducting polymers (LCP) ${ }^{1-5}$. SN is the main characteristic of laser action and in LCP it typically occurs at high excitation intensities and is accompanied by excitonic lifetime shortening ${ }^{4,5}$. The origin of $\mathrm{SN}$ has been independently attributed to lasing ${ }^{1,2}$, amplified spontaneous emission (ASE) $)^{3.4}$, and cooperative radiation or superfluorescence (SF) ${ }^{5}$. These nonlinear optical phenomena need very high optical gain. However, whereas both lasing and ASE are the result of stimulated emission (SE), SF is due to the build-up of a macroscopic optical dipole moment ensuing from cooperative interactions between the photogenerated excitons ${ }^{6}$. It has been noted previously that the emission pattern remains isotropic at all excitation intensities ${ }^{2,4.5}$, which is inconsistent with an anisotropic (directional) SE process?.

In this study we compare contributions to SN in LCP from both SF and SE and determine a criterion at which SE becomes dominant. We report directional SE, which was obtained in improved, nonscattering thin films of 2,5-dioctyloxy PPV [DOO-PPV] $]^{5}$. Using an excitation area in the shape of a narrow stripe, we obtained directional SE in the form of a narrow waveguided beam in DOO-PPV solutions and films. We demonstrate that SN in this case could be achieved by increasing the excitation area length, rather than the excitation intensity as in previous reports. The exponential gain coefficients were determined for different excitation intensities. We also observed gain saturation and estimated the optical loss coefficient for our films to be about $30 \mathrm{~cm}^{-1}$.

We investigated photopumped lasing using cylindrical microcavity structures formed by thin LCP films deposited on glass fibers. Two LCP were tested: derivatives of poly(p-phenylene-vinylene) [PPV], having the photoluminescence (PL) band in the red spectral range, and di-substituted polyacetylene (DPA $)^{8}$, having the PL band in the green. Multimode narrow laser lines $\left(-1.5 \mathrm{~cm}^{-1}\right)$ with low threshold intensities $(1 \mathrm{~nJ} /$ pulse) are demonstrated in these structures using pulsed photoexcitation. We note that low lasing threshold is an important criterion in developing electrically pumped plastic lasers. 


\section{EXPERIMENTAL}

For studies of SE in LCP films, DOO-PPV films with uniform thickness, $d$, ranging from $0.5 \mu \mathrm{m}$ to $4 \mu \mathrm{m}$ were slowly spin-coated from fresh chloroform solutions onto quartz substrates. The variation in $d$ was typically less than $5 \%$ over the film length of $1 \mathrm{~mm}$. The excitation source for DOO-PPV films was the frequency doubled output $(532 \mathrm{~nm})$ of a Nd:YAG regenerative laser amplifier producing $100 \mathrm{ps}$ pulses at $500 \mathrm{~Hz}$. The pump laser beam was focused on the polymer film using either a round lens for round spot excitation, or a cylindrical lens to obtain an excitation area in the form of a $100 \mu \mathrm{m}$ thin stripe. The round spot size could be varied from $30 \mu \mathrm{m}$ to $3 \mathrm{~mm}$. The length of the stripe-like excitation area could be varied from $100 \mu \mathrm{m}$ to $6 \mathrm{~mm}$ using a variable-width slit in front of the film, which could block parts of the pump beam. The polymer emission was collected from either the front or the side of the substrate and spectrally analyzed using either a 0.25 meter spectrometer or a 0.6 meter triple spectrometer. All experiments were performed in dynamic vacuum at room temperature.

In our studies of lasing in LCP films, we used soluble derivatives of both PPV and DPA: DOO-PPV and PDPA-nBu ${ }^{8}$, respectively. Cylindrically shaped thin polymer films were prepared by dipping commercially available optical fibers into saturated chloroform solutions. Thin polymer rings were consequently formed around the glass cylindrical core following the fast drying in air. The estimated thickness of the deposited polymer rings was about 2-3 $\mu \mathrm{m}$. The excitation source was the Nd:YAG regenerative laser amplifier producing 100ps pulses at a repetition rate of $100 \mathrm{~Hz}$. This laser light was either frequency doubled $(532 \mathrm{~nm})$ or tripled $(355 \mathrm{~nm})$ for pumping DOO-PPV or PDPA-nBu films, respectively. The pump beam was focused using a cylindrical lens into a $100 \mu \mathrm{m} \times 5 \mathrm{~mm}$ stripe perpendicular to the polymer coated fiber $(\varnothing 10-200 \mu \mathrm{m})$; thus only a small fraction of the pump light was absorbed by the polymer film. The pump beam polarization could be rotated to be either parallel or perpendicular to the fiber axis. The light emitted from the excited polymer ring was collected in the plane of the ring with a round lens and spectrally analyzed using a 0.6 meter triple spectrometer and a CCD array with spectral resolution of about $1 \AA$.

\section{RESULTS AND DISCUSSION}

Initial experiments with DOO-PPV films showed ${ }^{5}$ that the polymer emission remained isotropic at all excitation intensities, and no well-defined waveguiding was then observed. Similar results were obtained with other LCP films ${ }^{24}$. This could be attributed to poor film quality which consequently leads to strong light scattering inside the film and on its surface, so that the observed SN is isotropic. Therefore, optical confinement in such films is weak and a characteristic length of the non-linear emission process leading to $\mathrm{SN}$ is on the order of $d$. On the other hand, it can be argued that a SE process requires a substantially longer characteristic length', thus SF appears to be a more appropriate explanation for the isotropic SN. The onset of SN in LCP is usually characterized by a well-defined threshold excitation intensity, $\mathrm{I}_{0}$, or excitation power, $\mathrm{P}_{0}{ }^{1-5}$. Using the same DOO-PPV films that were used in ref. 5, we measured the dependence of $P_{0}$ on the diameter, $D$, of a round excitation spot as shown in Fig.1. It can be seen that $P_{o}$ is practically constant in the range of $100 \mu \mathrm{m}<D<400 \mu \mathrm{m}$, which cannot be explained with a simple SE process'. On the other hand, a SF process is governed by the total number of photogenerated excitons and thus is determined by the total excitation power ${ }^{10}$. In this case SF is a more suitable explanation, and from Fig.l we can estimate the cooperation length ${ }^{10}$ of excitons in DOO-PPV films to be about $400 \mu \mathrm{m}$.

The main features of SF, such as the intensity dependence, pulse shortening, time delay etc., can be described within a simple semi-classical approach using Maxwell-Bloch equations, first introduced by Bonifacio and Lugiato ${ }^{6}$. It can be shown then that in a mean-field approximation a system of excited, non-interacting emitters is described by a pendulum equation ${ }^{6}$ :

$$
\ddot{S}_{Z}+\left(\kappa+\frac{1}{2 T_{2}^{*}}\right) \dot{S}_{Z}=-\frac{g_{0}^{2}}{V} e^{-t / T_{2}^{*}}\left(2 S_{T}^{2}+4 A_{T}^{2} S_{Z}\right)
$$

where $S_{z}$ is the exciton population, $S_{T}$ describes the cooperative, macroscopic dipole moment of the system, $A_{T}$ is the photon number operator of the emitted electromagnetic field, $K$ is the radiation leakage rate out of the active volume $V, T_{2}{ }^{2}$ is the inhomogeneous dephasing time, and $\mathrm{g}_{0}$ is the coupling constant, which is proportional to the exciton oscillator strength. It can be seen that the right-hand side of Eq.(1) contains two driving terms: the macroscopic dipole moment of the excitons and their radiated electromagnetic field. The first term gives rise to cooperative radiation or SF, whereas the second term is the source of SE. Thus it becomes apparent that, in general, the resultant emission contains contributions from both SF and SE. 


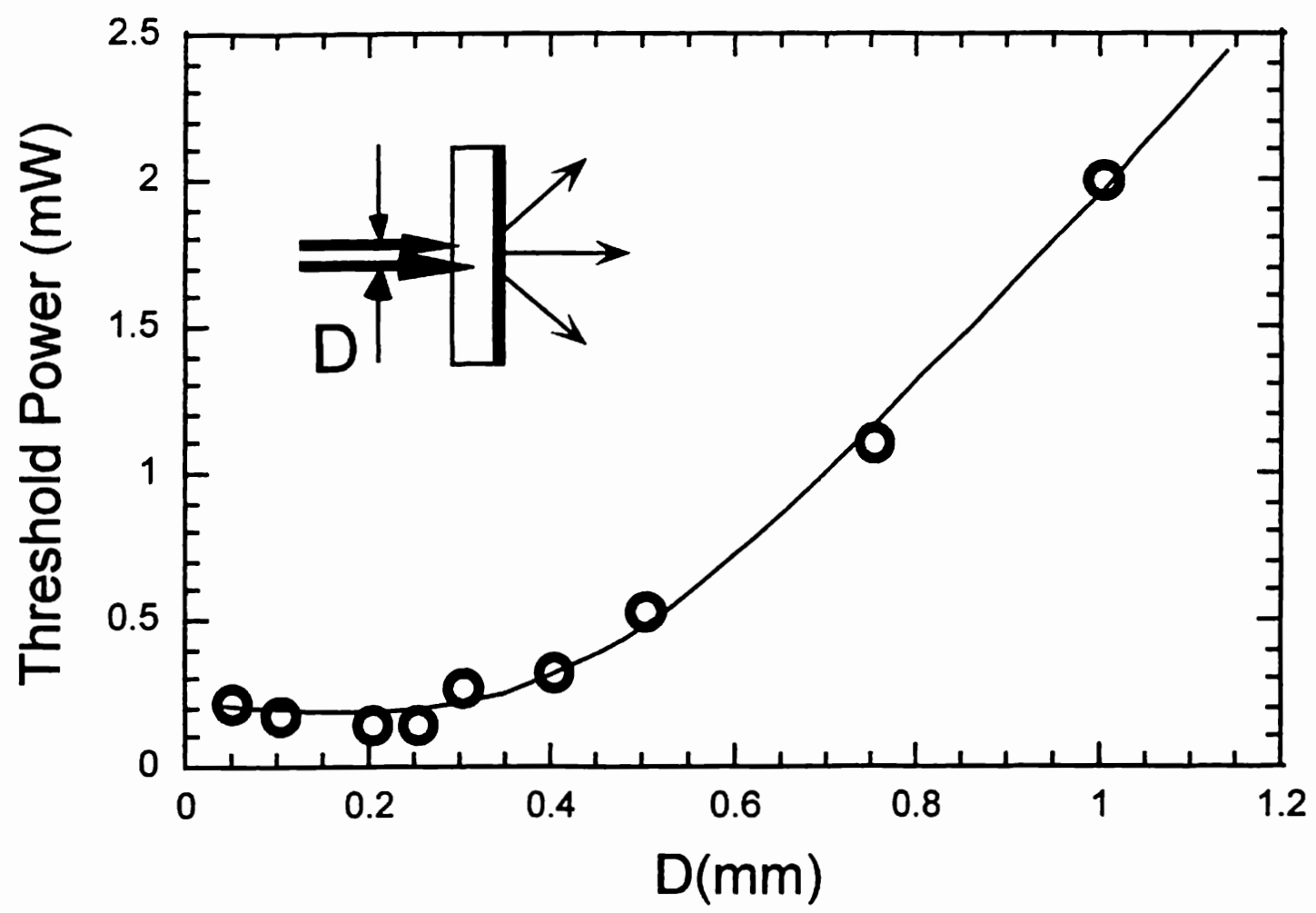

Fig.1. SN threshold power dependence on the excitation spot size in DOO-PPV films; the experimental schematic is given in the inset.

Whether this nonlinear emission is due to SF or SE is determined by the values of $T_{2}{ }^{\circ}$ and $\kappa \tau_{c}$. Here $\tau_{c}$ is the ArrechiCourtens cooperation time ${ }^{6}$ given by $\left(g_{0} \sqrt{N / V}\right)^{-1}$, where $N$ is the number of excitons. If $T_{2}{ }^{\circ}>1 / k$, then the balance between SF and SE is decided by the degree of optical confinement in the active volume, or the relative magnitude of $k$. Assuming that the characteristic length of SN in LCP is on the order of $d$, we find that this condition is well satisfied ${ }^{5}$. It can be seen that $\left(\kappa \tau_{c}\right)^{-1}$ is proportional to the number photons emitted via the SE process. Thus the condition $\kappa \tau_{c} \ll 1$ identifies a process where SE is dominant; on the other hand, $\kappa \tau_{c} \gg 1$ defines the case of "pure" SF ${ }^{6}$. Poor optical confinement, which was previously observed in LCP films, suggests that $K$ in this case is very high, so that emitted photons leave the system too fast to induce SE in the active volume and therefore $\kappa \tau_{c}>1$. As a result, pure SF may then be observed in DOO-PPV films for $D<400 \mu \mathrm{m}$.

Conditions for SE can be achieved by either decreasing $\kappa$ or increasing $\tau_{c}$. By diluting the DOO-PPV chromophore concentration in solution and thus decreasing $N / V$, we are able to increase $\tau_{c}$, so that $\tau_{c}>T_{2}{ }^{\circ}$, and subsequently suppress $\mathrm{SF}^{9,10}$. Fig.2(a) shows the emission spectra of dilute DOO-PPV solutions in chloroform, measured at various excitation intensities using transverse photoexcitation as shown in Fig.2(a), inset. It can be seen that SN in DOO-PPV solutions significantly differs from that in DOO-PPV films ${ }^{5}$. We measured the dependence of the emission peak intensity, $\mathrm{I}_{\mathrm{se}}$, on the excitation intensity, $I_{p}$, as shown in Fig.2(b). $I_{s e}$ grows exponentially with $I_{p}$, which is consistent with an ASE process ${ }^{7}$ described by

$$
I_{s e}=\beta \cdot\left(e^{(\gamma-\alpha) L}-1\right)
$$

where $\beta$ is a constant which depends on the excitation geometry ${ }^{7}$, and $\gamma$ and $\alpha$ are the optical gain and loss coefficients at the peak intensity wavelength $\lambda(\sim 590 \mathrm{~nm})$. Since $\gamma$ grows linearly with $I_{p}$ at relatively low intensities, we find that $\ln \left(I_{s e}\right) \propto I_{p}$ at large $I_{s e}$, in agreement with functional dependence in Fig.2(b).

Alternatively, we can achieve $\kappa \tau_{c} \ll 1$ by decreasing $\kappa$, which requires substantial improvement in the optical confinement inside the polymer film. Recently, we were able to improve the quality of our films and fabricated low 

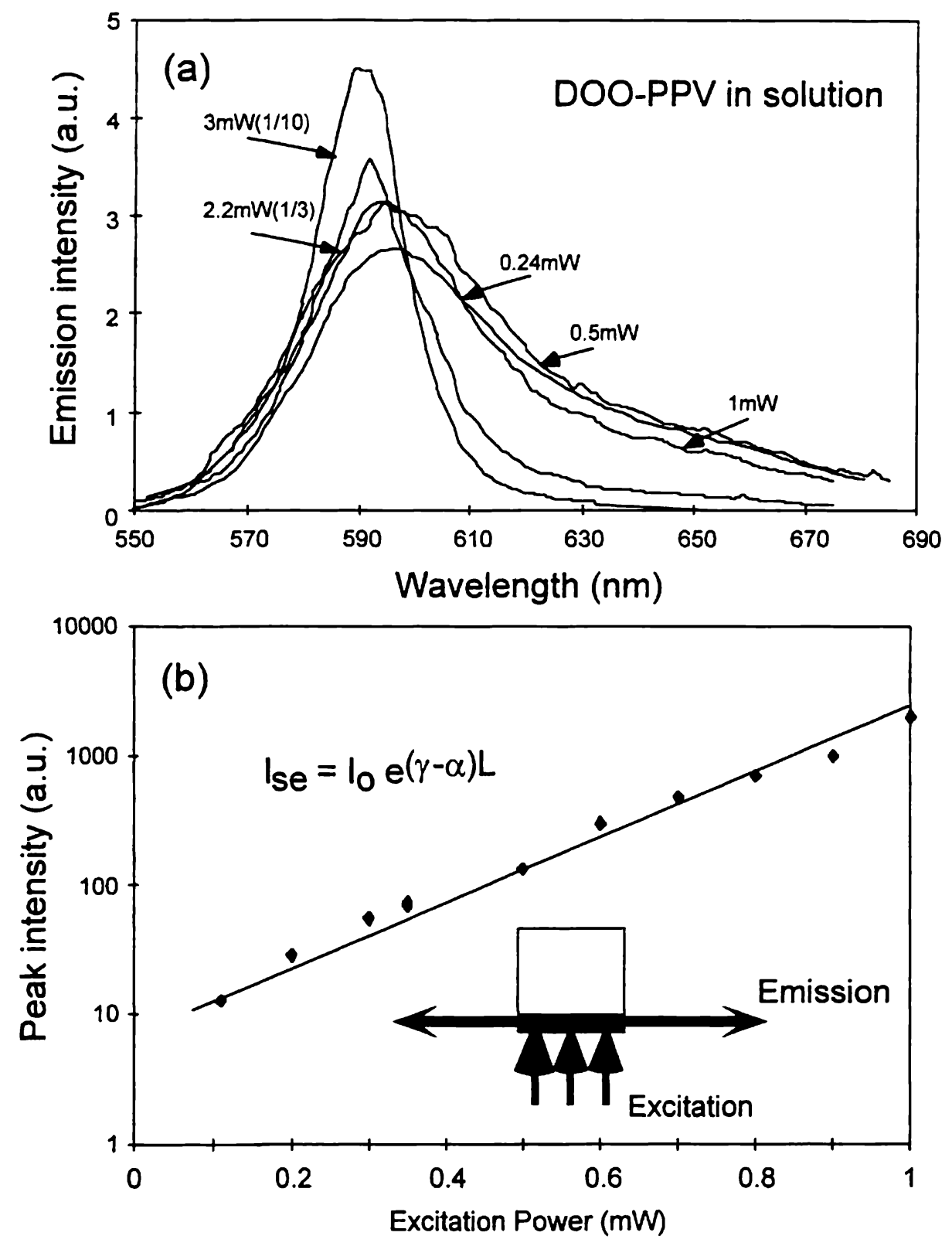

Fig.2. Normalized emission spectra at various excitation intensities in DOO-PPV solutions (a) and corresponding peak intensity dependence on $\mathrm{I}_{\mathrm{p}}(\mathrm{b})$; the experimental setup in shown in the inset.

scattering planar waveguides from DOO-PPV. As a result, a large portion of the polymer emission was optically confined inside the film by internal reflections. Using the refraction index of the substrate, $n_{s}=1.46$, and that of the DOO-PPV film, 
$\mathrm{n}_{\mathrm{p}}=1.7$, we estimate the fraction of emission which is waveguided or "trapped" inside the film to be $f=\sqrt{1-\left(\frac{n_{s}}{n_{p}}\right)^{2}}=0.51$.

The spectrally narrow SE $(\sim 8 \mathrm{~nm})$ from such films was observed only in the plane of the film, whereas the emission perpendicular to the film surface remained spectrally broad $(80 \mathrm{~nm})$, as shown in Fig.3. This indicates that SE is due to waveguided polymer emission which propagates alongside the film and thus experiences the largest gain. For our measurements of SE we used an excitation spot in the shape of a narrow stripe. As a result, SE was predominantly emitted along the axis of the stripe.

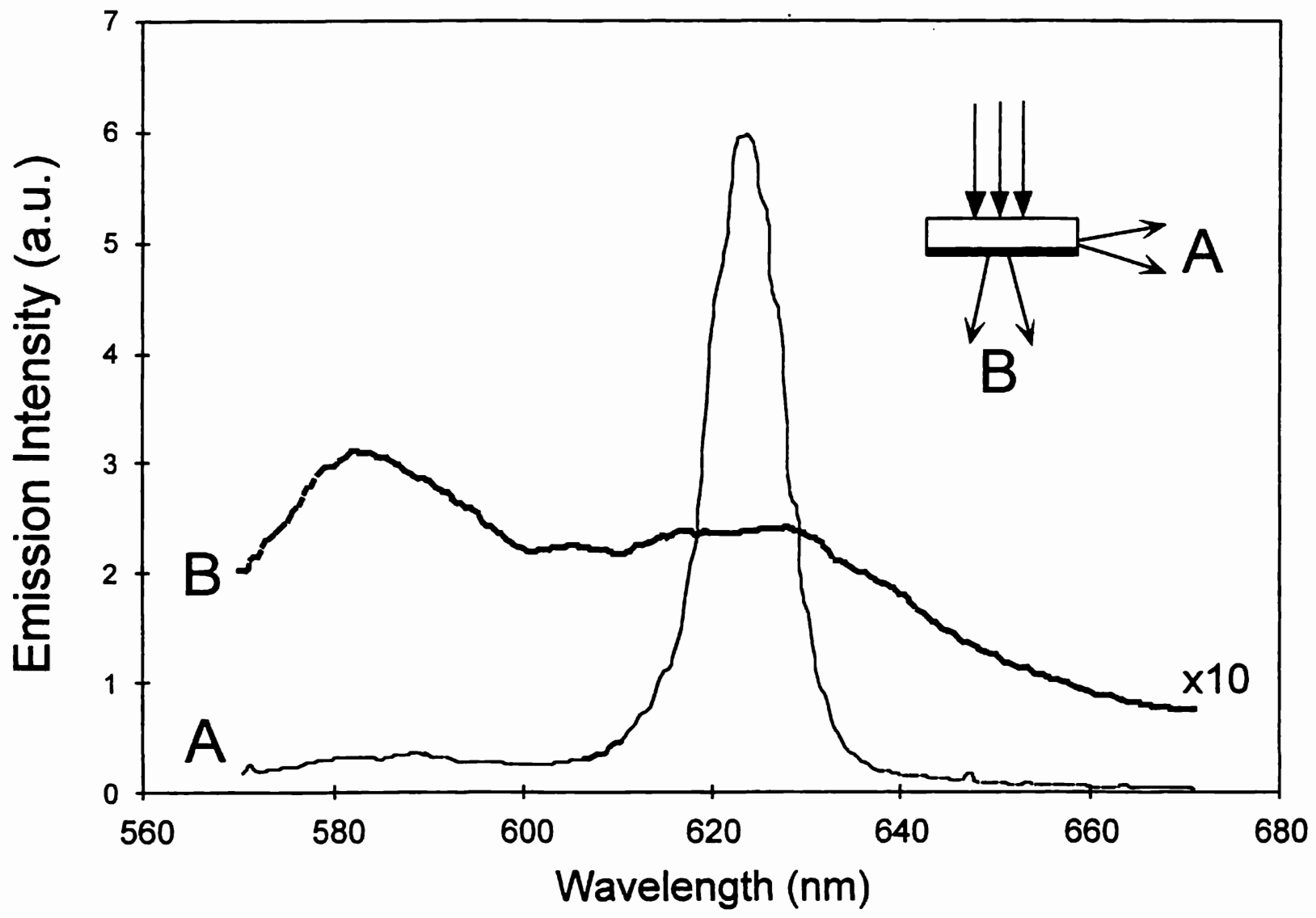

Fig.3. The emission spectra of improved DOO-PPV films measured in the directions parallel (A) and perpendicular (B) to the film surface at the same excitation intensity; emission intensity B is magnified by 10 . The inset illustrates the experimental setup.

Fig.4 shows SE spectra obtained by increasing either the excitation intensity $I_{p}$ (a) or the excitation stripe length $L$ (b). The results are virtually identical: in both cases the spectral narrowing of the polymer emission was observed above threshold values of $I_{0}$ and $L_{0}$, respectively. SE in this case can be modeled using the ASE approximation with cylindrical excitation and Eq.(2). ${ }^{7}$ We assume that $\gamma \propto \mathrm{I}_{\mathrm{p}}$ and $\alpha$ is constant. Since $\gamma$ depends on $\lambda$ and has a maximum at $\sim 630 \mathrm{~nm}, \mathrm{SE}$ at different $\lambda$ is amplified differently and $I_{s e}(630 \mathrm{~nm})$ experiences the maximum gain. Consequently, this nonlinear amplification leads to $S N$ when either $I_{p}$ or $L$ is increased.

We measured $I_{s e}$ dependence on $\mathrm{L}$ at different $I_{p}$, as shown in Fig.5(a). In accordance with Eq.(2), SE grows exponentially at small $\mathrm{L}$. This allows us to estimate the corresponding effective gain coefficients: $(\gamma-\alpha) \sim 70 \mathrm{~cm}^{-1}$ for $I_{p}=30$ $\mathrm{mW} / \mathrm{cm}^{2}$, and $\sim 40 \mathrm{~cm}^{-1}$ for $I_{p}=20 \mathrm{~mW} / \mathrm{cm}^{2}$. We thus obtain $\gamma \sim 3.4 \cdot I_{p}\left(\mathrm{~mW} / \mathrm{cm}^{2}\right)$ and $\alpha \sim 30 \mathrm{~cm}^{-1}$. However, as $L$ increases, 


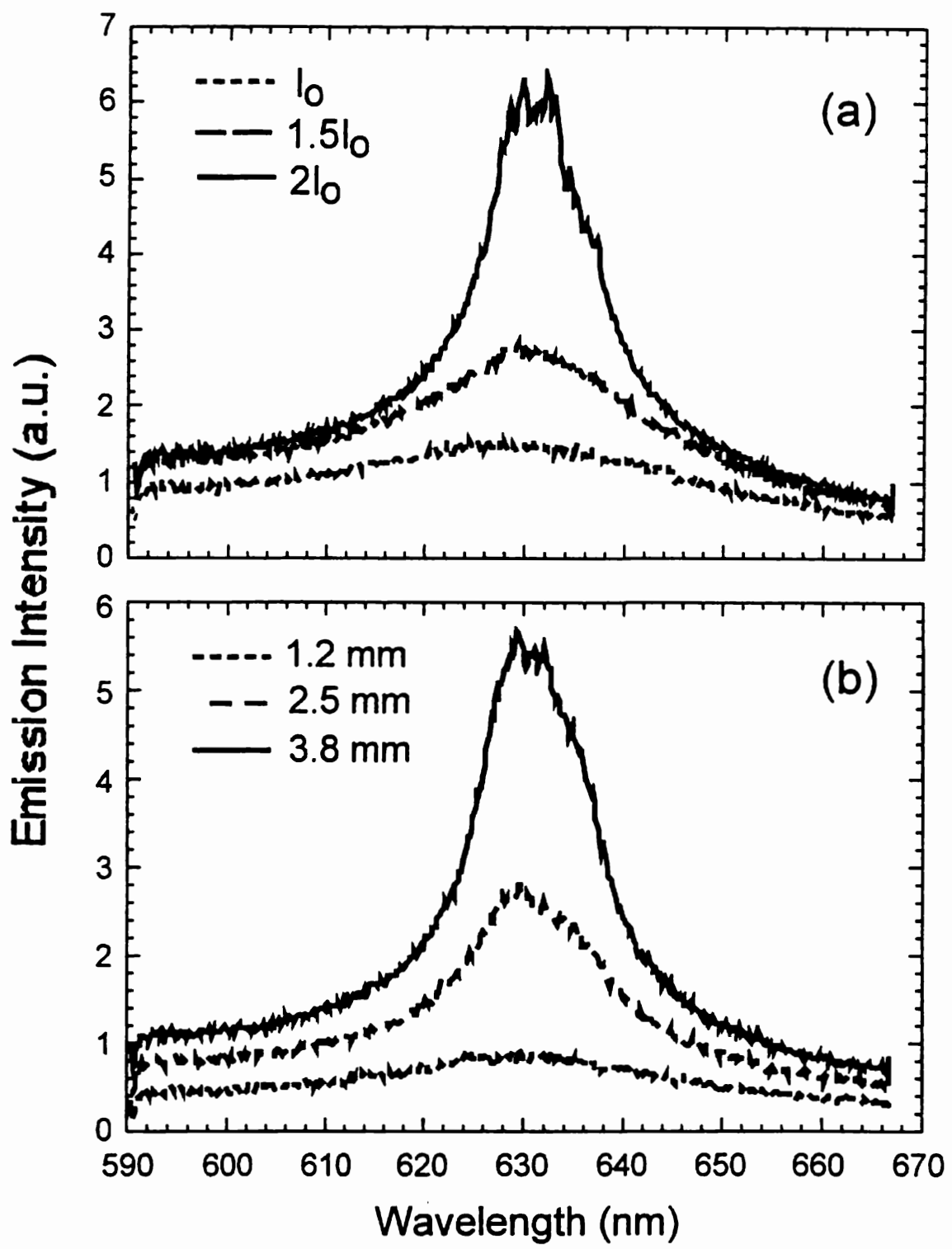

Fig.4. $S N$ in new DOO-PPV films with increasing $I_{p}$ at $L=2 m m$ (a) and with increasing $L$ (b).

so does $I_{\text {se, }}$, which eventually leads to gain saturation when $I_{\text {se }}$ approaches the saturation intensity. ${ }^{7}$ In the limit of the absorption limited gain saturation $I_{s e}$ stops growing altogether, as can be seen in Fig.5(a).

We can define the threshold for SE in DOO-PPV films by the onset of the nonlinear amplification of the $630 \mathrm{~nm}$ emission peak. From Eq.(2) the condition when the nonlinear SE peak at $630 \mathrm{~nm}$ becomes visible is then given by $(\gamma-\alpha) \mathrm{L}>$ 1 , which can be rewritten as follows:

$$
\gamma \geq \alpha+\frac{1}{\mathrm{~L}} \text {. }
$$

Fig.5(b) shows the threshold gain $\gamma_{t}$ measured at various $L ; \gamma_{t}$ was calculated from the measured threshold intensity and the previously determined relation between $I_{p}$ and $\gamma$. Using Eq.(3) we can model Fig.5(b) and calculate $\alpha$ at $\lambda=630 \mathrm{~nm}$ to be $-30 \mathrm{~cm}^{-1}$, which confirms our previous estimate. 

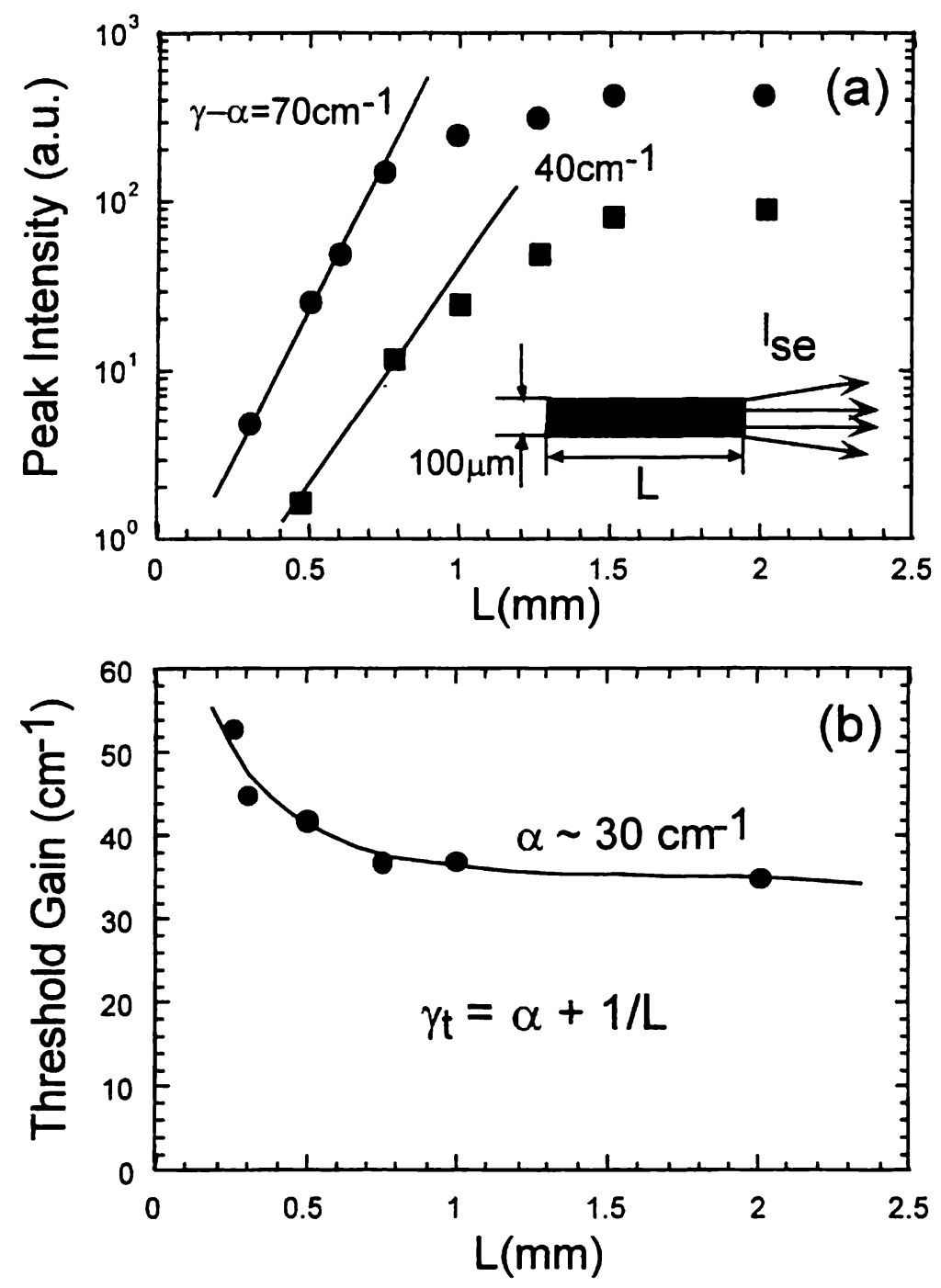

Fig.5. SE peak intensity dependence on $L$ for $I_{p}=30 \mathrm{~mW} / \mathrm{cm}^{2}$ (circles) and $20 \mathrm{~mW} / \mathrm{cm}^{2}$ (squares) (a), the inset shows the excitation area; SE threshold gain coefficient calculated for various $L(b)$.

It can be seen from Fig.5(b) that the SE threshold for $\mathrm{L}>1 \mathrm{~mm}$ is mainly determined by $\alpha$. This is an important implication for future plastic lasers. A similar threshold condition might occur in a laser where the cavity finesse $Q$, which determines the threshold for lasing, is limited by selfabsorption: $Q \leq Q_{a b s}=2 \pi / \alpha \lambda^{7.12}$. From the measured $\alpha$ we estimate $Q_{a b s}$ to be about 3000 , whereas $Q$ of a typical laser ranges from 100 to $1000 . Q_{a b s}$ thus determines the lowest attainable lasing threshold ${ }^{12}$. We expect that in our case $\alpha$ is due to both self-absorption and scattering, and thus should strongly vary from film to film. This means that it is feasible to further decrease $\alpha$ and lower the threshold for both ASE and lasing. 


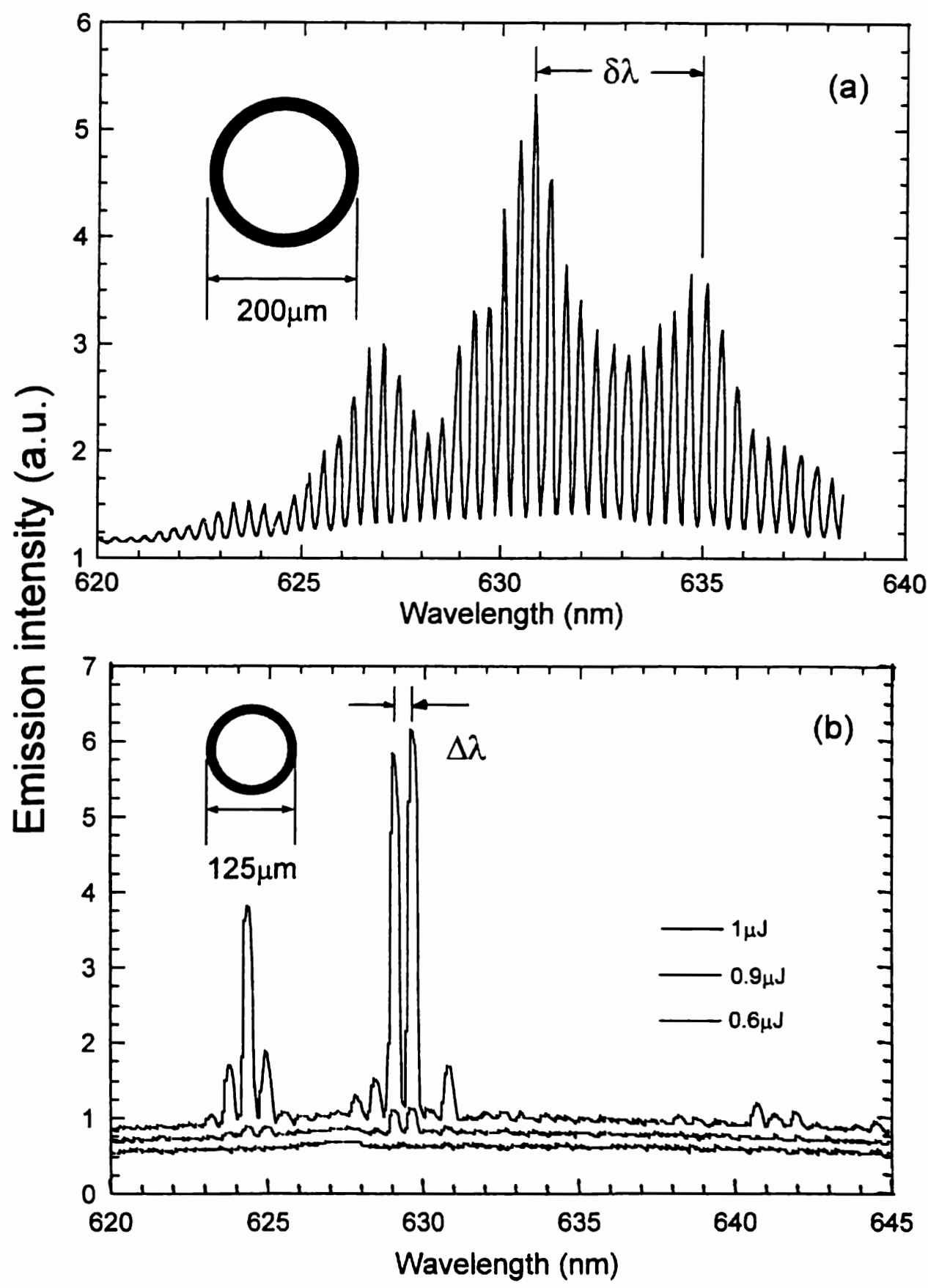

Fig.6. Emission spectra from DOO-PPV $\mu$ rings on a $200 \mu \mathrm{m}$ diameter fiber (a) and a $125 \mu \mathrm{m}$ diameter fiber (b).

We investigated photopumped lasing in LCP using cylindrical microcavity structures made out of thin films. In general, microcavities provide excellent coupling of spontaneous emission into lasing modes, high quality factors $Q$, and correspondingly low lasing thresholds ${ }^{13}$.The polymer self-assembled microstructures discussed here, i.e. cylindrical microrings, are very similar to those previously reported in literature ${ }^{14}$. In this work however we have exclusively used pristine conducting polymers as the lasing medium. Fig.6(a) shows a multimode lasing spectrum obtained from a DOO-PPV 
coated silica fiber with an outer diameter of $200 \mu \mathrm{m}$. The spectral peak intensity of the polymer ring laser is positioned at the maximum of optical gain, which in turn corresponds to the PL spectrum peak. The polymer film acts as a curved waveguide and forms a ring resonator. The laser emission occurs in the plane of the ring. The resonant wavelengths for the waveguided lasing modes are given by the following equation ${ }^{14}$ :

$$
m \lambda_{m}=\pi D n_{\text {eff }}
$$

where $m$ is an integer, $\lambda_{m}$ is the resonant wavelength, $D$ is the fiber diameter and $n_{\text {eff }}$ is the effective index of refraction in the cylindrical waveguide. The latter is determined by the thickness $d$, bulk refraction index of the polymer $\mathrm{n}_{\mathrm{p}} \sim 1.7$, " refraction index of the fiber $n_{g}=1.46$, and the radius of curvature $R=D / 2$. For substantially thick polymer films, as in this case, $n_{\text {eff }}$ is approximately given by $n_{\text {eff }} \approx n_{p}(l-d / D) \approx n_{p}$. Therefore, we can estimate the intermodal spacing as follows $\Delta \lambda=\lambda_{m}-\lambda_{m-1}=\frac{\lambda^{2}}{\pi D n_{\text {eff }}}=3.71 \AA$, which closely corresponds to the experimentally observed $\Delta \lambda$ in Fig.6(a) of $3.8 \AA$.

Similar results were obtained using $125 \mu \mathrm{m}$ diameter optical fibers coated with DOO-PPV [Fig.6(b)]. The observed $\Delta \lambda$ in this case is $6 \AA$, whereas $\Delta \lambda$ estimated from Eq.(4) is $5.94 \AA$. Fig.6(b) shows the corresponding emission spectra below and above the lasing threshold intensity, $\mathrm{l}_{0}$, indicating a sharp onset of the stimulated emission which is one of the main characteristic features attributed to lasers. The corresponding $I_{o}$ is about $3 \cdot 10^{4} \mathrm{~W} / \mathrm{cm}^{2}$. This intensity is about an order of magnitude lower than the previously reported threshold intensity for spectral narrowing in flat DOO-PPV films.

The polymer $\mu$ ring laser emission is linearly polarized. Its polarization is parallel to the fiber axis, which corresponds to TE modes. The measured polarization ratio $I_{11} / I_{\perp}$ of the ring laser emission above $I_{o}$ is greater than 100 . Here $I_{11}$ and $I_{\perp}$ are the emission intensities with polarizations parallel and perpendicular to the fiber axis, respectively. The background PL however is characterized by $I_{11} / I_{\perp}$ which is close to one. Furthermore, the polarization of the $\mu$ ring emission does not depend on the pump polarization. However, when the pump beam polarization is along the fiber (TE polarization), $\mathrm{I}_{\mathrm{o}}$ is approximately twice lower than that when the pump polarization is perpendicular to the fiber (TM polarization).

The thin ring resonator shown in the inset of Fig.6(b) is formed around the glass fibers which can support whispering gallery modes propagating along their cylindrical edges ${ }^{13}$. In the complete ring cylindrical resonator whispering modes are not dominant, since the waveguided modes are better confined. However, whispers are able to produce a low $Q$ feedback mechanism to modify $\mathrm{Q}_{\mathrm{cav}}$ of the primary waveguided modes in the polymer ring. $\mathrm{Q}_{\mathrm{cav}}$ increases if waveguided and whispering modes are in resonance, and $Q_{c a v}$ decreases if the corresponding resonant wavelengths are detuned from each

other. It can be shown ${ }^{12}$ that the associated modulation in $Q_{\text {cav }}$ has a period of $\delta \lambda=\Delta \lambda \frac{n_{\text {eff }}}{\Delta n_{\text {eff }}}$, where $\Delta n_{\text {eff }}=n_{\text {eff }}-n_{\text {eff }}$ is the difference between $\mathrm{n}_{\mathrm{eff}}$ of the waveguided and whispering modes, respectively.

It can be seen that equally spaced laser modes shown in Fig. 6 are periodically modulated in intensity. This additional intensity modulation could be explained by the variation in the quality factor $Q_{m}$ versus $\lambda_{m}$. The total $Q_{m}$ is given by

$$
\mathrm{Q}_{\mathrm{m}}{ }^{-1}=\mathrm{Q}_{\mathrm{abs}}{ }^{-1}+\mathrm{Q}_{\mathrm{scal}}{ }^{-1}+\mathrm{Q}_{\mathrm{cav}}{ }^{-1}
$$

where $Q_{a b s}=2 \pi / \alpha \lambda$ is the absorption limited quality factor, $Q_{s c a t}$ is determined by scattering losses due to imperfections inside the polymer film and on its surface, and $Q_{c a v}$ is the microcavity finesse determined only by its geometry. In case of cylindrical microcavities ${ }^{13} \mathrm{Q}_{\mathrm{cav}}$ is in the range of $10^{4}-10^{8}$. $\mathrm{Q}_{\text {sca }}$ strongly depends on the polymer film quality and in theory can be made very high; its dependence on $\lambda$ is slow and thus can be ignored. $Q_{a b s}$ is estimated from $\alpha(630 \mathrm{~nm}) \sim 30 \mathrm{~cm}^{-1}$ in our samples to be of order $10^{3}-10^{4}$. Therefore, we can approximate $Q_{m} \sim Q_{a b s}$. This approximation is confirmed by the independent estimates of $\mathrm{Q}_{\mathrm{m}}$ from the values of the cavity resonance half-width and $\mathrm{I}_{\mathrm{o}}$. The observed laser emission is mostly due to light tunneling through the outer film surface and scattering on imperfections. Thus, the lasing mode intensity is approximately given by $I_{m} \propto\left(Q_{c a v}^{-1}+Q_{s c a t}^{-1}\right) \cdot Q_{m}$. If $Q_{m}$ is wavelength dependent, then so is $I_{m}$. $Q_{c a v}$ in thin film ring resonators can be periodically modulated with period $\delta \lambda$, as has been shown above. Thus, from Fig. I(a) we can estimate $\Delta n_{\text {eff }}$ $\sim 0.1 \mathrm{n}_{\text {eff. }}$

We also built $\mu$ ring lasers using PDPA-nBu, which has a broad PL spectrum in the green spectral range peaking at $\sim 530 \mathrm{~nm}$. Fig.7(a) shows a multimode lasing spectrum of a PDPA $\mu$ ring formed around a $125 \mu \mathrm{m}$ diameter fiber. The distance between neighboring modes is about $4 \AA$, from which we estimate $n_{\text {eff }}$ to be about 1.75 for PDPA-nBu. Fig.7(b) shows the pumping intensity dependence of the spectrally integrated emission intensity. There is a very well defined $I_{0}$ marked by the kink in the slope. It indicates an increased directionality of the emission pattern, i.e., more light is emitted into the plane of the ring. This feature is also a salient characteristic of lasing. 

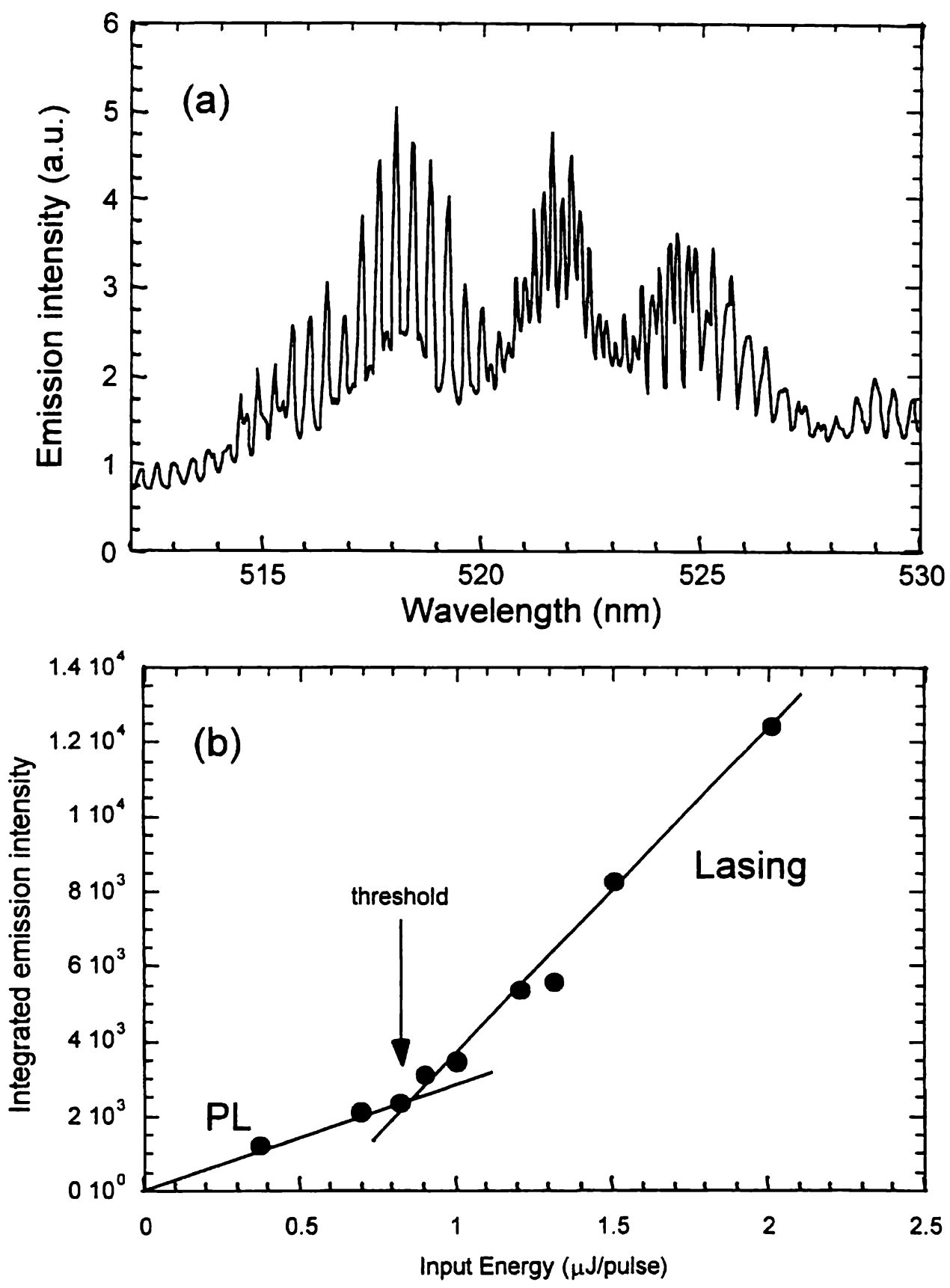

Fig.7. Emission spectra from PDPA-nBu $\mu$ ring on a $125 \mu \mathrm{m}$ fiber at $\sim 1 \mu \mathrm{J} /$ pulse (a) and the excitation intensity dependence of the $\mu$ ring laser emission intensity (b).

\section{CONCLUSIONS}

In conclusion, we showed that both cooperative radiation (SF) and stimulated emission (SE) contribute to the emission spectral narrowing in DOO-PPV films. We argued that SF is favored in thin films with poor optical confinement for 
excitation spot sizes smaller than the exciton cooperation length of about $400 \mu \mathrm{m}$. However, in DOO-PPV solutions and thin waveguides with good optical confinement we demonstrated directional SE. Spectral narrowing in this case was obtained by increasing either the excitation length or the excitation intensity. We found that the SE threshold in films in case of a large excitation area is mainly determined by the optical loss coefficient of about $30 \mathrm{~cm}^{-1}$. The SE intensity was found to rapidly increase above the threshold and quickly approach the saturation intensity, which in turn led to gain saturation. We demonstrated multimode lasing in cylindrical microcavities formed by thin films of various conducting polymers. These lasers are characterized by (1) drastic emission spectral narrowing, where a single mode linewidth is less than $1 \AA$, (2) well defined lasing threshold intensity, (3) increased directionality in the emission pattern, (4) high degree of polarization above the threshold, and (5) high Q of order $10^{4}$.

We thank Drs. W.Gellermann and D.Chinn for assistance with measurements. This work was done at the Utah Laser Institute and supported in part by the DOE grant no. FG-03-96 ER 45490.

\section{REFERENCES}

1. N.Tessler, G.J.Denton and R.H.Friend, Nature 382, 695 (1996).

2. F.Hide et al., Science 273, 1833 (1996); Chem. Phys. Lett. 256, 424 (1996).

3. H.J.Brouwer, V.V. Krasnikov, A.Hilberer, and G.Hadziioanou, Adv. Materials 8, 935 (1996).

4. G.H.Gelink, J.M.Warman, M.Remmers, and D.Neher, Chem. Phys. Lett. 265, 320 (1997).

5. S.V.Frolov et al., Jpn. J. Appl. Phys. 35, L1371 (1996); Synth. Metals 84/1-3, 471-474 (1997); Phys. Rev. Lett. 78, 729 (1997).

6. R. Bonifacio and L.A. Lugiato, Phys. Rev. A 11, 1507 (1975).

7. A.Yariv, Quantum Electronics (Wiley, New York, 1975).

8. K.Tada et al., Jpn. J. Appl. Phys. 35, L1138 (1996).

9. S.V.Frolov, Z.V.Vardeny, and K.Yoshino, Cooperative and Stimulated Emission in Poly(p-phenylene-vinylene) Thin Films and Solutions. (to be published)

10. Q.H.F.Vrehen and H.M.Gibbs, in Dissipative Systems in Quantum Optics, v.27 of Topics in Current Physics (ed. R.Bonifacio, Springer, Berlin, 1982).

11. The refractive index of DOO-PPV films was found to vary between 1.7 and 1.9; for our estimates we used the lowest value.

12. S.V. Frolov, M.Shkunov, Z.V.Vardeny, and K.Yoshino, Phys. Rev. B 56, 4363 (1997).

13. Y.Yamamoto and R.Slusher, Physics Today 46, 66 (1993); J.C.Knight, H.S.T.Driver, and G.N.Robertson, J.Opt. Soc. Am. B 11, 2046 (1994).

14. H.P.Weber and R.Ulrich, Appl. Phys. Lett. 19, 38 (1971); M.Kuwata-Gonokami et al., Opt. Lett. 20, 2093 (1995).

15. M.Heiblum and J.H.Harris, IEEE J. Quantum Electronics QE-11, 75 (1975). 